\title{
MINIMIZING ENERGY AMONG HOMOTOPIC MAPS
}

\author{
PENGZI MIAO
}

Received 6 May 2003

\begin{abstract}
We study an energy minimizing sequence $\left\{u_{i}\right\}$ in a fixed homotopy class of smooth maps from a 3-manifold. After deriving an approximate monotonicity property for $\left\{u_{i}\right\}$ and a continuous version of the Luckhaus lemma (Simon, 1996) on $S^{2}$, we show that, passing to a subsequence, $\left\{u_{i}\right\}$ converges strongly in $W^{1,2}$ topology wherever there is small energy concentration.
\end{abstract}

2000 Mathematics Subject Classification: 53C43, 58E20.

1. Introduction. Let $\phi: M \rightarrow N$ be a continuous map between two compact Riemannian manifolds. In general, there may not exist a harmonic map homotopic to $\phi$ (see [2]). Hence, a map $u$ that minimizes energy among all smooth maps homotopic to $\phi$ may not exist. However, it is still a basic question to understand the analytical property of a minimizing sequence. If the domain $M$ is a compact surface, it is known to experts that any minimizing sequence that converges weakly indeed converges strongly in $W^{1,2}$ topology away from a finite number of points, where energy concentrates and bubble forms (see [3, 7]). If the domain is of higher dimension, B. White showed that the infimum of the energy functional over the homotopy class of $\phi$ is determined only by the restriction of $\phi$ to a 2-skeleton of $M$ (see [8]). It is our goal in this paper to apply White's result to derive a similar theorem for 3-manifolds.

THEOREM 1.1. Let $\phi: M \rightarrow N$ be a smooth map between two compact Riemannian manifolds without boundary. Assume that $M$ has dimension 3. Then there exists a constant $\epsilon_{0}=\epsilon_{0}(M, N)>0$ such that, for any sequence of maps $\left\{u_{i}\right\}$ which minimizes energy among all smooth maps homotopic to $\phi$ and converges weakly in $W^{1,2}(M, N)$, if

$$
\liminf _{i \rightarrow \infty} \frac{1}{\sigma} \int_{B_{\sigma}(x)}\left|d u_{i}\right|^{2} d V \leq \epsilon_{0}
$$

then $\left\{u_{i}\right\}$ converges strongly in $W^{1,2}\left(B_{\sigma / 4}(x), N\right)$.

As an application of Theorem 1.1, we prove a partial regularity result for the weak limit of an energy minimizing sequence.

COROLLARY 1.2. Let $\phi: M \rightarrow N$ be a smooth map between two compact Riemannian manifolds without boundary, where $M$ has dimension 3. Let $\left\{u_{i}\right\}$ be a sequence of maps which minimizes energy among all smooth maps homotopic to $\phi$. Suppose that $\left\{u_{i}\right\}$ converges weakly to some $u \in W^{1,2}(M, N)$; then there exists a closed set $\Sigma \subset M$ with finite 
1-dimensional Hausdorff measure such that $u$ is a smooth harmonic map from $M \backslash \Sigma$ to $N$. In particular, $u$ is a weakly harmonic map from $M$ to $N$.

We remark that the dimension restriction of the domain space comes only from the lemma proved in Section 4. If a similar lemma could be established on a general sphere $S^{n-1} \subset \mathbb{R}^{n}$, the rest of the argument in this paper would imply that Theorem 1.1 holds for arbitrary dimension $n$ with the small energy concentration assumption (1.1) replaced by

$$
\liminf _{i \rightarrow \infty} \frac{1}{\sigma^{n-2}} \int_{B_{\sigma}(x)}\left|d u_{i}\right|^{2} d V \leq \epsilon_{0}
$$

2. Preliminaries. Let $\left(M^{3}, g\right)$ and $\left(N^{m}, h\right)$ be compact Riemannian manifolds of dimensions 3 and $m$. We assume that $M$ and $N$ have no boundary. By the Nash embedding theorem, it is convenient to regard $N$ as isometrically embedded in some Euclidean space $\mathbb{R}^{K}$. We define

$$
W^{1,2}(M, N)=\left\{u \in W^{1,2}\left(M, \mathbb{R}^{K}\right) \mid u(x) \in N \text { a.e. } x \in M\right\},
$$

where $W^{1,2}\left(M, \mathbb{R}^{K}\right)$ is the separable Hilbert space of maps $u: M \rightarrow \mathbb{R}^{K}$ whose component functions are $W^{1,2}$ Sobolev functions on $M$. We note that $W^{1,2}(M, N)$ inherits both strong and weak topologies from $W^{1,2}\left(M, \mathbb{R}^{K}\right)$. Moreover, it is a strongly closed set with the property that, for any $C>0$,

$$
\left\{u \in W^{1,2}(M, N) \mid\|u\|_{W^{1,2}} \leq C\right\}
$$

is weakly compact in $W^{1,2}(M, N)$ (see [4]).

For any $u \in W^{1,2}(M, N)$, the energy of $u$ is defined by

$$
E(u)=\int_{M} \operatorname{Tr}_{g}\left(u^{*} h\right) d V=\int_{M}\left|d u^{2}\right| d V
$$

where $u^{*} h$ is the pullback of $h$ by $u$ and $d V$ is the volume measure determined by $g$ on $M$.

Let $C^{\infty}(M, N) \subset W^{1,2}(M, N)$ be the space of smooth maps. For any $\phi \in C^{\infty}(M, N)$, we define

$$
\begin{gathered}
\mathscr{F}_{\phi}=\left\{u \in C^{\infty}(M, N) \mid u \text { is homotopic to } \phi\right\}, \\
E_{\phi}=\inf \left\{E(u) \mid u \in \mathscr{F}_{\phi}\right\} .
\end{gathered}
$$

The following result, which is due to White [8], gives a fundamental characterization of $E_{\phi}$.

WHITE'S THEOREM. Let $\mathscr{F}_{\phi}^{(2)}=\left\{u \in C^{\infty}(M, N) \mid u\right.$ is 2 -homotopic to $\left.\phi\right\}$, where two continuous maps $v$ and $w$ are said to be 2-homotopic if their restrictions to the 2dimensional skeleton of some triangulation of $M$ are homotopic. Then

$$
\inf \left\{E(u) \mid u \in \mathscr{F}_{\phi}^{(2)}\right\}=E_{\phi} .
$$


Let $\left\{u_{i}\right\} \subset \mathscr{F}_{\phi}$ be an arbitrary sequence which minimizes the energy functional, that is,

$$
\lim _{i \rightarrow \infty} E\left(u_{i}\right)=E_{\phi}
$$

Then the above theorem suggests that $\left\{u_{i}\right\}$ is also a minimizing sequence in $\mathscr{F}_{\phi}^{(2)}$. This fact is very useful since it allows more competitors to be compared with $u_{i}$.

By the weak compactness of bounded sets in $W^{1,2}(M, N)$, we may assume that, passing to a subsequence, $\left\{u_{i}\right\}$ converges weakly in $W^{1,2}(M, N)$, strongly in $L^{2}(M, N)$, and pointwise almost everywhere to some $u \in W^{1,2}(M, N)$, which has the property that

$$
E(u) \leq \lim _{i \rightarrow \infty} E\left(u_{i}\right)=E_{\phi} .
$$

Moreover, by the Riesz representation theorem, we know that there exists a Radon measure $\mu$ on $M$ so that

$$
\left|d u_{i}\right|^{2}(x) d V \rightarrow \mu .
$$

Throughout the paper, we use $c_{1}, c_{2}, c_{3}, \ldots$ to denote constants depending only on $(M, g)$ and $(N, h)$.

3. Approximate monotonicity of $\left\{\left|d u_{i}(x)\right|^{2} d V\right\}$. Given a $C^{1}$ vector field $X$ on $M$, we let $\left\{F_{t}\right\}$ denote the one-parameter group of diffeomorphism on $M$ generated by $X$. For any $v \in W^{1,2}(M, N)$, we define $E_{v}(t, X)=E\left(v \circ F_{t}\right)$, where $v \circ F_{t}(x)=v\left(F_{t}(x)\right)$. The first variation formula for the energy functional (see [4]) then gives that

$$
\frac{d}{d t} E_{v}(t, X)=\int_{M}\left\langle v^{*} h,-g^{\prime}(t)+\frac{1}{2}\left\{\operatorname{Tr}_{g}(t) g^{\prime}(t)\right\} g(t)\right\rangle_{g(t)} d V(t)
$$

where $v^{*} h$ is the pullback of $h$ by $v, g(t)=F_{-t}^{*}(g)$, and $d V(t)$ is the volume measure determined by $g(t)$. In particular, at $t=0$, we have that

$$
\frac{d}{d t} E_{v}(0, X)=\int_{M}\left\langle v^{*} h, \mathscr{L}_{X} \mathcal{g}-\frac{1}{2}\left\{\operatorname{Tr}_{\mathfrak{g}}\left(\mathscr{L}_{X} \mathcal{g}\right)\right\} \mathfrak{g}\right\rangle_{g} d V
$$

The following lemma says that, for large $n, u_{n}$ is "almost stationary" with respect to a large class of domain variations.

LEMMA 3.1. Given $\Lambda>0$, let $V_{\Lambda}=\left\{C^{1}\right.$ vector field $X$ with $\left.\|X\|_{C^{1}} \leq \Lambda\right\}$. Then

$$
\sup _{X \in V_{\Lambda}}\left\{\frac{d}{d t} E_{n}(0, X)\right\} \rightarrow 0 \text { as } n \rightarrow \infty \text {, }
$$

where $E_{n}(t, X)=E_{u_{n}}(t, X)$.

Proof. Let $\sigma_{0}$ be a sufficiently small positive constant depending only on $(M, g)$ such that for any geodesic ball $B_{\sigma}\left(x_{0}\right) \subset M$ with $\sigma \leq \sigma_{0}$ and any geodesic normal coordinate chart $\left\{x^{1}, x^{2}, x^{3}\right\}$ in $B_{\sigma}\left(x_{0}\right)$, all the eigenvalues of the matrix $\left[g_{i j}(x)\right]_{3 \times 3}$ lie 
in $[1 / 2,2]$ for each $x \in B_{\sigma}\left(x_{0}\right)$. With such a choice of $\sigma$, we have that

$$
\begin{aligned}
\int_{B_{\sigma}\left(x_{0}\right)}|d v|^{2} d V & =\sum_{\alpha=1}^{K} \int_{B_{\sigma}\left(x_{0}\right)} \frac{\partial v^{\alpha}}{\partial x^{i}} \frac{\partial v^{\alpha}}{\partial x^{j}} g^{i j}(x) d V \\
& \geq \frac{1}{2} \sum_{\alpha=1}^{K} \int_{B_{\sigma}\left(x_{0}\right)} \sum_{i=1}^{3}\left|\frac{\partial v^{\alpha}}{\partial x^{i}}\right|^{2}(x) d x
\end{aligned}
$$

for any $v \in W^{1,2}\left(M, \mathbb{R}^{K}\right)$, where $d x$ denotes the Lebesgue measure in $\mathbb{R}^{3}$.

To prove the lemma, we first consider $V_{\Lambda, \sigma}$ instead of $V_{\Lambda}$, where $\sigma \leq \sigma_{0}$ and

$$
V_{\Lambda, \sigma}=\left\{X \in V_{\Lambda} \mid \operatorname{support}\{X\} \subset B_{\sigma}\left(x_{0}\right) \text { for some } x_{0} \in M\right\} \text {. }
$$

For any $X \in V_{\Lambda, \sigma}$, we write

$$
\begin{gathered}
G(t)=-g^{\prime}(t)+\frac{1}{2}\left\{\operatorname{Tr}_{g(t)} g^{\prime}(t)\right\} g(t), \\
H^{i j}(t, x)=G_{k l}(t, x) g^{i k}(t, x) g^{j l}(t, x) \sqrt{\operatorname{det}\left(g_{i j}(t, x)\right)} .
\end{gathered}
$$

It follows from (3.1) that

$$
\frac{d}{d t} E_{m}(t, X)-\frac{d}{d t} E_{m}(0, X)=\int_{B_{\sigma}\left(x_{0}\right)}\left(u_{m}^{*} h\right)_{i j}(x)\left\{H^{i j}(t, x)-H^{i j}(0, x)\right\} d x,
$$

where

$$
\left(u_{m}^{*} h\right)_{i j}(x)=\sum_{\alpha=1}^{K} \frac{\partial u_{m}^{\alpha}}{\partial x^{i}}(x) \frac{\partial u_{m}^{\alpha}}{\partial x^{j}}(x) .
$$

Hence,

$$
\begin{aligned}
& \left|\frac{d}{d t} E_{m}(t, X)-\frac{d}{d t} E_{m}(0, X)\right| \\
& \quad \leq 6 \sum_{i, j=1}^{3}\left(\sup _{x \in B_{\sigma}\left(x_{0}\right)}\left|H^{i j}(t, x)-H^{i j}(0, x)\right|\right) \cdot\left(\int_{B_{\sigma}\left(x_{0}\right)}\left|d u_{m}\right|^{2} d V\right)
\end{aligned}
$$

by the Cauchy-Schwartz inequality and (3.4). We note that $H^{i j}(t, x)$ is a known function of $\left\{g_{i j}(t, x)\right\}$ and $\left\{(d / d t) g_{i j}(t, x)\right\}$, while $g(t, x)=F_{-t}^{*} g(x)$ and $(d / d t) g(t, x)=$ $F_{-t}^{*}\left(\mathscr{L}_{X} g\right)(x)$. Since $\|X\|_{C^{1}} \leq \Lambda$, it follows from the standard ODE theory that, for any $\epsilon>0$, there exists $t_{0}$ depending only on $\epsilon, \Lambda$, and $g$ so that, for any $t \in\left[-t_{0}, t_{0}\right]$, we have that $\|g(t)-g\|_{C^{1}} \leq \epsilon$, hence $\left|H^{i j}(t, x)-H^{i j}(0, x)\right| \leq C \epsilon$ for some constant $C$ depending only on the algebraic expression of $H^{i j}$.

Now assume that the lemma is not true for $V_{\Lambda, \sigma}$; then there exist $\delta_{0}>0$, a sequence of $\left\{X_{k}\right\} \subset V_{\Lambda, \sigma}$, and a subsequence $\left\{u_{i_{k}}\right\}$ of $\left\{u_{i}\right\}$ such that

$$
\left|\frac{d}{d t} E_{i_{k}}\left(0, X_{k}\right)\right|>\delta_{0}
$$


Our above analysis then shows that there exists $t_{0}=t_{0}\left(\delta_{0}, \mathcal{g}, \Lambda, E_{\phi}\right)$ such that

$$
\left|\frac{d}{d t} E_{i_{k}}\left(t, X_{k}\right)\right|>\frac{1}{2} \delta_{0} \quad \forall t \in\left[-t_{0}, t_{0}\right] .
$$

Since $\lim _{k \rightarrow \infty} E\left(u_{i_{k}}\right)=E_{\phi}$, we conclude that, for some $k$ large enough and some $t \in$ $\left[-t_{0}, t_{0}\right], E\left(u_{i_{k}} \circ F_{t}\right)<E_{\phi}-(1 / 4) \delta_{0} t_{0}$, which is a contradiction to the fact $u_{i_{k}} \circ F_{t} \in \mathscr{F}_{\phi}$ and the definition of $E_{\phi}$.

To replace $V_{\Lambda, \sigma}$ by $V_{\Lambda}$, we can simply apply a partition of unity argument considering that $(d / d t) E_{n}(0, X)$ is linear in $X$. Hence, the lemma is proved.

Now we are ready to derive an approximate monotonicity property for $\left\{u_{i}\right\}$. Let $\xi(t)$ be any $C^{1}$ decreasing function on $[0,+\infty)$ whose support lies in $[0,1]$. We fix $x_{0} \in M$ and let $\left\{x^{1}, x^{2}, x^{3}\right\}$ be a geodesic normal coordinate chart in $B_{\sigma}\left(x_{0}\right)$. For $0<\rho<\sigma \leq \sigma_{0}$ and $x \in B_{\sigma}\left(x_{0}\right)$, we define $X_{\rho}(x)=\xi(|x| / \rho) x^{i}\left(\partial / \partial x^{i}\right)$ and view $X_{\rho}$ as a vector field defined globally on $M$. It is easily checked that $\left\|X_{\rho}\right\|_{C^{1}} \leq \Lambda$ for some constant $\Lambda=\Lambda(\xi)>0$. Thus Lemma 3.1 implies that there exists a sequence $\left\{\kappa_{i}\right\}$ depending on $\Lambda(\xi)$ but not on $\rho$ such that

$$
\left|\frac{d}{d t} E_{i}\left(0, X_{\rho}\right)\right| \leq \kappa_{i}, \quad \lim _{i \rightarrow \infty} \kappa_{i}=0
$$

A direct calculation shows that

$$
\begin{aligned}
\frac{d}{d t} E_{i}\left(0, X_{\rho}\right)= & \operatorname{error}(\rho)+(-1) \int_{B_{\sigma}\left(x_{0}\right)} \xi\left(\frac{|x|}{\rho}\right)\left|d u_{i}\right|^{2} d V \\
& +(-1) \int_{B_{\sigma}\left(x_{0}\right)} \xi^{\prime}\left(\frac{|x|}{\rho}\right)\left(\frac{|x|}{\rho}\right)\left|d u_{i}\right|^{2} d V \\
& +2 \int_{B_{\sigma}\left(x_{0}\right)} \xi^{\prime}\left(\frac{|x|}{\rho}\right)\left(\frac{|x|}{\rho}\right)\left|\frac{\partial u_{i}}{\partial v}\right|^{2} d V
\end{aligned}
$$

where $v=\left(x^{i} /|x|\right)\left(\partial / \partial x^{i}\right)$, $|\operatorname{error}(\rho)| \leq \bar{c} \rho^{2}\left(\int_{B_{\sigma}\left(x_{0}\right)}\left|d u_{i}\right|^{2} d V\right)$, and $\bar{c}=\bar{c}(\xi, g)$. We then define

$$
E_{i}(\rho)=\frac{1}{\rho} \int_{B_{\sigma}\left(x_{0}\right)} \xi\left(\frac{|x|}{\rho}\right)\left|d u_{i}\right|^{2} d V
$$

It follows from (3.12) and (3.13) that

$$
E_{i}^{\prime}(\rho)+\bar{c} \int_{B_{\sigma}\left(x_{0}\right)}\left|d u_{i}\right|^{2} d V \geq-\kappa_{i} \frac{1}{\rho^{2}}
$$

which gives that

$$
E_{i}(\tau) \leq E_{i}(\rho)+\bar{c}(\rho-\tau) \int_{B_{\sigma}\left(x_{0}\right)}\left|d u_{i}\right|^{2} d V+\kappa_{i}\left(\frac{1}{\tau}-\frac{1}{\rho}\right)
$$

for any $0<\tau<\rho<\sigma$. Hence, we have proved the following proposition. 
Proposition 3.2. For any $C^{1}$ decreasing function $\xi(t)$ with support in $[0,1]$, there exists a sequence $\left\{\kappa_{i}\right\}$ such that $\lim _{i \rightarrow 0} \kappa_{i}=0$ and

$$
\begin{aligned}
\frac{1}{\tau} \int_{B_{\sigma}\left(x_{0}\right)} \xi\left(\frac{|x|}{\tau}\right)\left|d u_{i}\right|^{2} d V \leq & \frac{1}{\rho} \int_{B_{\sigma}\left(x_{0}\right)} \xi\left(\frac{|x|}{\rho}\right)\left|d u_{i}\right|^{2} d V \\
& +\bar{c}(\rho-\tau) \int_{B_{\sigma}\left(x_{0}\right)}\left|d u_{i}\right|^{2} d V+\kappa_{i}\left(\frac{1}{\tau}-\frac{1}{\rho}\right)
\end{aligned}
$$

for any $x_{0} \in M$ and any $0<\tau<\rho<\sigma \leq \sigma_{0}$. Here $\left\{\kappa_{i}\right\}$ is independent of $\rho$ and $\tau$, and $\bar{c}$ is a constant depending only on $\xi$ and $g$.

Letting $i$ go to $\infty$, we have the following "monotonicity" formula for the limiting measure $\mu$.

Corollary 3.3. For any $C^{1}$ decreasing function $\xi(t)$ with its support in $[0,1]$,

$$
\frac{1}{\tau} \int_{B_{\sigma}\left(x_{0}\right)} \xi\left(\frac{|x|}{\tau}\right) d \mu \leq \frac{1}{\rho} \int_{B_{\sigma}\left(x_{0}\right)} \xi\left(\frac{|x|}{\rho}\right) d \mu+\bar{c} \mu\left(B_{\sigma}\left(x_{0}\right)\right)
$$

for any $x_{0} \in M, 0<\tau<\rho<\sigma \leq \sigma_{0}$, and some constant $\bar{c}=\bar{c}(\xi, g)$. Choosing $\xi$ to be 1 on $[0,1 / 2]$,

$$
\frac{1}{\tau} \mu\left(B_{\tau}\left(x_{0}\right)\right) \leq \frac{2}{\rho} \mu\left(B_{\rho}\left(x_{0}\right)\right)+\bar{c} \mu\left(B_{\sigma}\left(x_{0}\right)\right)
$$

for any $0<2 \tau<\rho<\sigma \leq \sigma_{0}$, where $\bar{c}=\bar{c}(g)$.

As an application of this "monotonicity" property of $\mu$, we show that $u$ can be well approximated by smooth maps into $N$ from the region where $\left\{u_{i}\right\}$ has small energy concentration.

Proposition 3.4. There exists a number $\epsilon_{1}$ depending only on $M$ and $N$ such that if $\mu\left(B_{\sigma}\left(x_{0}\right)\right) / \sigma<\epsilon_{1}$, then there exists a sequence of smooth maps $\left\{u_{\tau}\right\}_{0<\tau<\tau_{0}}$ from $B_{\sigma / 2}\left(x_{0}\right)$ to $N$ such that $\lim _{\tau \rightarrow 0}\left\|u_{\tau}-u\right\|_{W^{1,2}\left(B_{\sigma / 2}\left(x_{0}\right)\right)}=0$.

Proof. We use the idea in [5] to mollify $u$. Let $\varphi: \mathbb{R}^{3} \rightarrow \mathbb{R}^{+}$be a smooth radial mollifying function so that support $(\varphi) \subset B_{1}$ and $\int_{\mathbb{R}^{3}} \varphi d x=1$. Assume that $\mu\left(B_{\sigma}\left(x_{0}\right)\right) / \sigma<$ $\epsilon_{1}$ for some $\epsilon_{1}$ to be determined later; by Corollary 3.3, we have that

$$
\frac{\mu\left(B_{\tau}(y)\right)}{\tau} \leq 4 \frac{\mu\left(B_{\sigma / 2}(y)\right)}{\sigma}+\bar{c} \sigma \epsilon_{1} \leq 4 \frac{\mu\left(B_{\sigma}\left(x_{0}\right)\right)}{\sigma}+\bar{c} \sigma \epsilon_{1} \leq 5 \epsilon_{1}
$$

for any $y \in B_{\sigma / 2}\left(x_{0}\right)$ and $0<2 \tau<\sigma / 2$ provided $\bar{c} \sigma \leq 1$. Now define $u^{\top}(y)=$ $\left(1 / \tau^{3}\right) \int_{B_{\tau}(y)} \varphi(|y-z| / \tau) u(z) d z$ inside a normal coordinate chart around $x_{0}$; we can apply a version of the Poincare inequality to assert that

$$
\frac{1}{\tau^{3}} \int_{B_{\tau}(y)}\left|u(x)-u^{\top}(y)\right|^{2} d x \leq c_{2} \frac{1}{\tau} \int_{B_{\tau}(y)}|d u|^{2} d x \leq c_{2} \frac{\mu\left(B_{\tau}(y)\right)}{\tau},
$$

where the last inequality holds because of the lower semicontinuity of energy with respect to weak convergence. It follows from (3.20) and (3.21) that $u^{\top}(y)$ lies near 
many values of $u(z)$ for $z \in B_{\tau}(y)$. In particular, we see that

$$
\operatorname{dist}\left(u^{\top}(y), N\right) \leq c_{3} \epsilon_{1}^{1 / 2} .
$$

Let $O_{\varepsilon}$ be a $\varepsilon$-tubular neighborhood of $N$ in $\mathbb{R}^{K}$, and let $\Phi: O_{\varepsilon} \rightarrow N$ denote the smooth nearest point projection map. We see that if $c_{3} \epsilon_{1}^{1 / 2}<\varepsilon$, then $u^{(\tau)}(y) \in \mathcal{O}_{\varepsilon}$ for all $y \in$ $B_{\sigma / 2}\left(x_{0}\right)$. Hence, we can define a smooth map $u_{\tau}: B_{\sigma / 2}\left(x_{0}\right) \rightarrow N$ by $u_{\tau}(y)=\Phi \circ u^{\top}(y)$. Since $u^{\tau}(y)$ is the standard mollification of $u$ by $\varphi$ with a scaling factor $\tau$, we see immediately that $\lim _{\tau \rightarrow 0}\left\|u_{\tau}-u\right\|_{W^{1,2}\left(B_{\sigma / 2} x_{0}\right)}=0$.

4. A continuous version of Luckhaus lemma. In this section, we use $\nabla(\cdot)$ to denote the gradient operator on $S^{2} \subset \mathbb{R}^{3}$ and $d \omega$ to denote the Euclidean surface measure on $S^{2}$. For a map $u$ defined on a cylinder $[a, b] \times S^{2}$, we use $\nabla_{x} u, \nabla_{t} u$ to denote the partial $x$, $t$ gradient of $u$, where $(t, x) \in[a, b] \times S^{2}$. The following technical lemma, which may be viewed as a continuous version of the 2-dimensional Luckhaus lemma (see [6]) in the study of energy minimizing maps, will help us construct comparison maps in the proof of the main theorem.

LEMMA 4.1. Assume that $N \subset \mathbb{R}^{K}$ is an isometrically embedded compact manifold. Then there exists $\epsilon_{2}=\epsilon_{2}(N)>0$ such that if $v, w \in W^{1,2}\left(S^{2}, N\right) \cap C^{0}\left(S^{2}, N\right)$ and

$$
\int_{S^{2}}|\nabla v|^{2} d \omega \leq \epsilon_{2}, \quad \int_{S^{2}}|\nabla w|^{2} d \omega \leq \epsilon_{2},
$$

then for all $\beta>0$, there exists $\eta=\eta\left(\epsilon_{2}, \beta\right)>0$, where $\eta$ does not depend on the choice of $v$ and $w$, such that if

$$
\int_{S^{2}}|v-w|^{2} d \omega<\eta
$$

then there exist $\beta^{\prime} \in[0, \beta)$ and $v^{\prime} \in W^{1,2}\left(\left[0, \beta^{\prime}\right] \times S^{2}, N\right) \cap C^{0}\left(\left[0, \beta^{\prime}\right] \times S^{2}, N\right)$ with properties that

$$
\begin{gathered}
v^{\prime}(0, x)=v(x), \quad v^{\prime}\left(\beta^{\prime}, x\right)=w(x), \\
\int_{\left[0, \beta^{\prime}\right] \times S^{2}}\left|\nabla_{(t, x)} v^{\prime}\right|^{2} d \omega d t \leq \beta .
\end{gathered}
$$

Proof. Let $v, w \in W^{1,2}\left(S^{2}, N\right) \cap C^{0}\left(S^{2}, N\right)$ such that (4.1) holds for some $\epsilon_{2}$ to be determined later. Let $\varphi: \mathbb{R}^{2} \rightarrow \mathbb{R}^{+}$be a smooth radial mollifying function so that $\operatorname{support}(\varphi) \subset B_{1}$ and $\int_{\mathbb{R}^{2}} \varphi d x=1$. For any $0<h \ll \pi / 2$ and any $(t, x) \in(0, h] \times S^{2}$, we define

$$
v(t, x)=\int_{S^{2}} v(y) \varphi^{t}(\operatorname{dist}(x, y)) d \omega(y),
$$

where $\operatorname{dist}(x, y)$ represents the sphere distance between $x$ and $y$ on $S^{2}$ and $\varphi^{t}(r)=$ $\left(1 / t^{2}\right) \varphi(r / t)$. Let $O_{2 \varepsilon}$ be a $2 \varepsilon$-tubular neighborhood of $N$ in $\mathbb{R}^{K}$; by the argument used in the proof of Proposition 3.4, we know that, if we choose $\epsilon_{2}=\epsilon_{2}(N)$ to be sufficiently small, then $v(t, x) \in O_{\varepsilon}$ for all $(t, x) \in(0, h] \times S^{2}$. (We note that the monotonicity of the 
energy of $v$, which is crucial in the proof of Proposition 3.4, is automatically satisfied in this case because the domain of $v$ is of 2-dimensional.) Since $v$ is continuous on $S^{2}$, we have that

$$
\lim _{(t, z) \rightarrow(0, x)} v(t, z)=v(x)
$$

Thus $v(t, x)$ is a continuous map on the closed cylinder $[0, h] \times S^{2}$ with $v(0, x)=v(x)$. On the other hand, if $B_{\sigma}(z)$ is a geodesic ball with a normal coordinate chart such that $x \in B_{\sigma / 2}(z)$, then for $0<h=h\left(\epsilon_{2}\right) \ll 1$, we have that

$$
v(t, x)=\int_{S^{2}} v(y) \varphi^{t}(\operatorname{dist}(x, y)) d \omega(y) \approx \int_{B_{\sigma}(0) \subset \mathbb{R}^{2}} v(x-t y) \varphi(|y|) d y,
$$

which implies that

$$
\begin{aligned}
& \left|\nabla_{x} v(t, x)\right|^{2} \leq c_{4} \int_{S^{2}}|\nabla v(y)|^{2} \varphi^{t}(\operatorname{dist}(x, y)) d \omega(y)+\epsilon_{2}, \\
& \left|\nabla_{t} v(t, x)\right|^{2} \leq c_{4} \int_{S^{2}}|\nabla v(y)|^{2} \varphi^{t}(\operatorname{dist}(x, y)) d \omega(y)+\epsilon_{2}
\end{aligned}
$$

by the Cauchy-Schwartz inequality. Then it follows from (4.7) that

$$
\int_{[0, h] \times S^{2}}\left|\nabla_{(t, x)} v(t, x)\right|^{2} d \omega(x) d t \leq c_{5} h\left(\int_{S^{2}}|\nabla v(y)|^{2} d \omega(y)+\epsilon_{2}\right)
$$

by the Fubini theorem. Similarly, we define $w(t, x):[l+h, l+2 h] \times S^{2} \rightarrow \mathbb{O}_{\varepsilon}$ by

$$
w(t, x)=\int_{S^{2}} w(y) \varphi^{(l+2 h-t)}(\operatorname{dist}(x, y)) d \omega(y)
$$

for some $l$ determined later.

Now we want to connect $v(h, x)$ and $w(l+h, x)$ on $[h, l+h] \times S^{2}$. We first estimate $|v(h, x)-w(l+h, x)|$ pointwise. It follows from the definition and the Cauchy-Schwartz inequality that

$$
\begin{aligned}
|v(h, x)-w(l+h, x)| & =\int_{S^{2}}|v(y)-w(y)| \varphi^{h}(\operatorname{dist}(x, y)) d \omega(y) \\
& \leq c_{6} \frac{1}{h}\left(\int_{S^{2}}|v(y)-w(y)|^{2} d \omega(y)\right)^{1 / 2} .
\end{aligned}
$$

We define $z(t, x)$ on $[h, l+h] \times S^{2}$ to be

$$
z(t, x)=\left(\frac{t-h}{l}\right) w(l+h, x)+\left(\frac{l+h-t}{l}\right) v(h, x) .
$$

Then (4.7) and (4.10) imply that

$$
\begin{aligned}
\int_{[h, l+h] \times S^{2}}\left|\nabla_{(t, x)} z(t, x)\right|^{2} d \omega(x) d t \leq & c_{7} l\left\{\int_{S^{2}}\left[|\nabla v|^{2}+|\nabla w|^{2}\right] d \omega+2 \epsilon_{2}\right\} \\
& +c_{7} \frac{1}{l} \frac{1}{h^{2}} \int_{S^{2}}|v(y)-w(y)|^{2} d \omega(y) .
\end{aligned}
$$


Now we consider

$$
\tilde{v}= \begin{cases}v(t, x), & 0 \leq t \leq h \\ z(t, x), & h \leq t \leq l+h \\ w(t, x), & l+h \leq t \leq l+2 h\end{cases}
$$

Clearly, $\tilde{v} \in C^{0} \cap W^{1,2}\left([0, l+2 h] \times S^{2}, \mathbb{R}^{K}\right)$. Furthermore,

$$
\begin{gathered}
\int_{[0, l+2 h] \times S^{2}}\left|\nabla_{(t, x)} \tilde{v}(t, x)\right|^{2} d \omega d t \leq \\
c_{8} \frac{1}{l} \frac{1}{h^{2}} \int_{S^{2}}|v(y)-w(y)|^{2} d \omega(y) \\
+c_{8}(h+l) \epsilon_{2} .
\end{gathered}
$$

For any $\beta>0$, we first choose $h=h\left(\beta, \epsilon_{2}\right)$ and $l=l\left(\beta, \epsilon_{2}\right)$ such that

$$
l+2 h<\beta, \quad c_{8}(h+l) \epsilon_{2}<\frac{\beta}{2},
$$

then we let $\|v-w\|_{L^{2}\left(S^{2}\right)}<\eta$, where $\eta=\eta\left(l, h, \beta, \epsilon_{2}\right)$ is so small that

$$
c_{8} \frac{1}{l} \frac{1}{h^{2}} \eta<\frac{\beta}{2}, \quad c_{6} \frac{1}{h} \eta^{1 / 2}<\varepsilon .
$$

It follows from (4.10) and (4.14) that $\tilde{v}(t, x) \in \mathrm{O}_{2 \varepsilon}$ for all $(t, x) \in[0, l+2 h] \times S^{2}$ and the total energy of $\tilde{v}$ is bounded by $\beta$. To get $v^{\prime}$ finally, we compose $\tilde{v}$ with the nearest point projection map $\Phi: \mathbb{O}_{2 \varepsilon} \rightarrow N$. Hence, the lemma is proved.

5. Proof of Theorem 1.1. Throughout this section, we fix a geodesic ball $B_{\sigma}\left(x_{0}\right)$, where

$$
\frac{1}{\sigma} \mu\left(B_{\sigma}\right)=\lim _{i \rightarrow \infty} \frac{1}{\sigma} \int_{B_{\sigma}}\left|d u_{i}\right|^{2} d V<\epsilon_{0}
$$

for some $\epsilon_{0}$ to be determined. For each $\tau$, we let $B_{\tau}$ denote $B_{\tau}\left(x_{0}\right)$.

Assume that $\epsilon_{0}<\epsilon_{1}$; Proposition 3.4 implies that there exists a sequence $\left\{v_{i}\right\} \subset$ $C^{\infty}\left(B_{\sigma / 2}, N\right)$ such that

$$
\lim _{i \rightarrow \infty}\left\|v_{i}-u\right\|_{W^{1,2\left(B_{\sigma / 2}\right)}}=0
$$

We then choose $\rho \in(\sigma / 4, \sigma / 2)$ such that $\left.u\right|_{\partial B_{\rho}},\left.u_{i}\right|_{\partial B_{\rho}} \in W^{1,2}\left(\partial B_{\rho}, N\right)$,

$$
\begin{gathered}
\lim _{i \rightarrow \infty}\left\|v_{i}-u\right\|_{W^{1,2}\left(\partial B_{\rho}\right)}=0, \\
\lim _{i \rightarrow \infty}\left\|u_{i}-u\right\|_{L^{2}\left(\partial B_{\rho}\right)}=0, \\
\int_{\partial B_{\rho}}|d u|^{2} d \Sigma \leq c_{9} \frac{1}{\sigma} \int_{B_{\sigma}}|d u|^{2} d V, \\
\liminf _{i \rightarrow \infty} \int_{\partial B_{\rho}}\left|d u_{i}\right|^{2} d \Sigma \leq c_{9} \frac{1}{\sigma} \lim _{i \rightarrow \infty} \int_{B_{\sigma}}\left|d u_{i}\right|^{2} d V,
\end{gathered}
$$


where $d \Sigma$ is the induced surface measure on $\partial B_{\rho} \subset M$. After fixing a geodesic normal coordinate chart centered at $x_{0}$, we may also view $\left.u\right|_{\partial B_{\rho}},\left.u_{i}\right|_{\partial B_{\rho}},\left.v_{i}\right|_{\partial B_{\rho}}$ as defined on the Euclidean sphere $S_{\rho}$ with radius $\rho$; then we have that

$$
\begin{gathered}
\int_{S_{\rho}}|\nabla u|^{2} d \omega_{\rho} \leq c_{10} \int_{\partial B_{\rho}}|d u|^{2} d \Sigma, \\
\int_{S_{\rho}}\left|\nabla u_{i}\right|^{2} d \omega_{\rho} \leq c_{10} \int_{\partial B_{\rho}}\left|d u_{i}\right|^{2} d \Sigma, \\
\int_{S_{\rho}}\left|\nabla v_{i}\right|^{2} d \omega_{\rho} \leq c_{10} \int_{\partial B_{\rho}}\left|d v_{i}\right|^{2} d \Sigma,
\end{gathered}
$$

where $d \omega_{\rho}$ represents the Euclidean surface measure on $S_{\rho}$. Now we choose $\epsilon_{0}<$ $\min \left\{\epsilon_{1},\left(c_{9} c_{10}\right)^{-1} \epsilon_{2}\right\}$ and we claim that

$$
\lim _{i \rightarrow \infty} \int_{B_{\rho}}\left|d u_{i}\right|^{2} d V=\int_{B_{\rho}}|d u|^{2} d V
$$

We remark that, once (5.5) is established, it will readily imply that

$$
\lim _{i \rightarrow \infty}\left\|u_{i}-u\right\|_{W^{1,2}\left(B_{\rho}\right)}=0
$$

by the fact that $\left\{u_{i}\right\}$ converges to $u$ weakly and the standard Hilbert space theories.

Assume that (5.5) does not hold; we have that

$$
\lim _{i \rightarrow \infty} \int_{B_{\rho}\left(x_{0}\right)}\left|d u_{i}\right|^{2} d V>\int_{B_{\rho}\left(x_{0}\right)}|d u|^{2} d V+2 \delta
$$

for some $\delta>0$. The idea for the rest of the proof is to construct a sequence of comparison maps which are almost $v_{i}$ inside $B_{\rho}$ and $u_{i}$ outside $B_{\rho}$. For that purpose, we need to connect $u_{i}$ and $v_{i}$ on the boundary of $B_{\rho}$ using Lemma 4.1. We first note that (5.3) imply that there exists a subsequence $\left\{u_{i_{k}}\right\},\left\{v_{i_{k}}\right\}$ such that

$$
\begin{gathered}
\int_{S_{\rho}}\left|\nabla u_{i_{k}}\right|^{2} d \omega_{\rho}<\epsilon_{2}, \quad \int_{S_{\rho}}\left|\nabla v_{i_{k}}\right|^{2} d \omega_{\rho}<\epsilon_{2} \quad \forall k, \\
\lim _{k \rightarrow \infty}\left\|v_{i_{k}}-u_{i_{k}}\right\|_{L^{2}\left(S_{\rho}\right)}=0 .
\end{gathered}
$$

We then consider $\bar{u}_{i_{k}}(\omega)=u_{i_{k}}(\rho \omega)$ and $\bar{v}_{i_{k}}(\omega)=v_{i_{k}}(\rho \omega)$, where $\omega$ denotes the point on $S^{2}$. It follows from (5.8) and Lemma 4.1 that for all $\beta>0$, there exist $k_{0}=k_{0}\left(\beta, \epsilon_{2}\right)$ and $\beta^{\prime}=\beta^{\prime}\left(\beta, \epsilon_{2}\right)<\beta$ such that for all $k>k_{0}$, there exists $\bar{w}_{k} \in W^{1,2} \cap C^{0}\left(\left[0, \beta^{\prime}\right] \times S^{2}, N\right)$ such that

$$
\begin{gathered}
\bar{w}_{k}(0, x)=\bar{u}_{i_{k}}(x), \\
\bar{w}_{k}\left(\beta^{\prime}, x\right)=\bar{v}_{i_{k}}(x), \\
\int_{\left[0, \beta^{\prime}\right] \times S^{2}}\left|\nabla_{(t, x)} \bar{w}_{k}\right|^{2} d \omega d t \leq \beta .
\end{gathered}
$$


Next, we use polar coordinates to transplant $\bar{w}_{k}$ to the shell region between $S_{\rho}$ and $S_{\left(1-\beta^{\prime}\right) \rho}$ by defining $w_{k}((1-t) \rho \omega)=\bar{w}_{k}(t, \omega)$. Rescaling $v_{i_{k}}(x)$ on $B_{\rho}$ to $v_{i_{k}}^{\prime}(x)=$ $v_{i_{k}}^{\prime}(r \omega)=v_{i_{k}}\left(r \omega /\left(1-\beta^{\prime}\right)\right)$ on $B_{\left(1-\beta^{\prime}\right) \rho}$, we then have that

$$
\begin{gathered}
w_{k}(x)=u_{i_{k}}(x), \quad x \in \partial B_{\rho}, \\
w_{k}(x)=v_{i_{k}}^{\prime}(x), \quad x \in \partial B_{\left(1-\beta^{\prime}\right) \rho}, \\
\int_{B_{\rho} \backslash B_{\left(1-\beta^{\prime}\right) \rho}}\left|d w_{k}\right|^{2} d V \leq c_{10} \rho \beta .
\end{gathered}
$$

Now we consider a new sequence $\left\{\hat{u}_{k}\right\} \subset W^{1,2} \cap C^{0}(M, N)$ given by

$$
\hat{u}_{k}= \begin{cases}u_{i_{k}}, & x \notin B_{\rho}, \\ w_{k}, & x \in B_{\rho} \backslash B_{\left(1-\beta^{\prime}\right) \rho}, \\ v_{i_{k}}^{\prime}, & x \in B_{\left(1-\beta^{\prime}\right) \rho} .\end{cases}
$$

First, we note that the fact that $\hat{u}_{k}=u_{i_{k}}$ outside $B_{\rho}$ and $\pi_{2}\left(S^{3}\right)=0$ implies that $\hat{u}_{k}$ is 2-homotopic to $u_{i_{k}}$. Second, we have the following energy estimate:

$$
\begin{aligned}
E\left(\hat{u}_{k}\right) & =\int_{M \backslash B_{\rho}}\left|d u_{i_{k}}\right|^{2} d V+\int_{B_{\rho} \backslash B_{\left(1-\beta^{\prime}\right) \rho}}\left|d w_{k}\right|^{2} d V+\int_{B_{\left(1-\beta^{\prime}\right) \rho}}\left|d v_{k}^{\prime}\right|^{2} d V \\
& \leq E\left(u_{i_{k}}\right)-\int_{B_{\rho}}\left|d u_{i_{k}}\right|^{2} d V+c_{10} \rho \beta+c\left(\beta^{\prime}\right) \int_{B_{\rho}}\left|d v_{i_{k}}\right|^{2} d V
\end{aligned}
$$

where $c\left(\beta^{\prime}\right)$ is the supremum of the Jacobian of the scaling diffeomorphism from $B_{\left(1-\beta^{\prime}\right) \rho}$ to $B_{\rho}$, which satisfies $\lim _{\beta^{\prime} \rightarrow 0} c\left(\beta^{\prime}\right)=1$ with the convergence only depending on $(M, g)$. We now fix $\beta$ such that

$$
\beta<\left(c_{10} \rho\right)^{-1} \delta, \quad\left|c\left(\beta^{\prime}\right)-1\right| \int_{B_{\rho}\left(x_{0}\right)}|d u|^{2} d V<\frac{\delta}{2}
$$

Letting $k \rightarrow \infty$, we then have that

$$
\limsup _{k \rightarrow \infty} E\left(\hat{u}_{k}\right) \leq E_{\phi}-\frac{\delta}{2}
$$

Finally, we note that the fact that $\hat{u}_{k} \in W^{1,2} \cap C^{0}(M, N)$ implies that $\hat{u}_{k}$ can be well approximated in $W^{1,2}$ norm by smooth maps from $M$ to $N$ which are homotopic to $\hat{u}_{k}$. One way to see this is to consider the standard mollification of $\hat{u}_{k}$ into $\mathbb{R}^{K}$, where the uniform continuity of $\hat{u}_{k}$ on $M$ will guarantee that the image of the mollification will be inside a tubular neighborhood of $N$. Composing it with the nearest point projection map, we then have the desired approximation. Hence, we know that there exists another sequence $\left\{\tilde{u}_{k}\right\} \subset C^{\infty}(M, N)$ such that $\tilde{u}_{k}$ is homotopic to $\hat{u}_{k}$ and

$$
\limsup _{k \rightarrow \infty} E\left(\tilde{u}_{k}\right)=\limsup _{k \rightarrow \infty} E\left(\hat{u}_{k}\right)<E_{\phi}-\frac{\delta}{2} .
$$


Since $\hat{u}_{k}$ and $u_{i_{k}}$ are 2-homotopic, we know that $\left\{\tilde{u}_{k}\right\} \subset \mathscr{F}_{\phi}^{(2)}$. Thus (5.15) gives a contradiction to the fact that $E_{\phi}=\inf \left\{E(u) \mid u \in \mathscr{F}_{\phi}^{(2)}\right\}$ by White's theorem. Therefore, (5.5) holds and Theorem 1.1 is proved.

Next, we prove Corollary 1.2 on the partial regularity of the weak limit of $\left\{u_{i}\right\}$. We recall the following $\epsilon$-regularity theorem for stationary harmonic maps obtained by Bethuel [1].

BETHUEL'S THEOREM. There exists a number $\epsilon_{3}=\epsilon_{3}(M, N)>0$ such that if $u$ : $B_{\sigma}\left(x_{0}\right) \subset M \rightarrow N$ is a stationary harmonic map and $(1 / \sigma) \int_{B_{\sigma}\left(x_{0}\right)}|d u|^{2} d V \leq \epsilon_{3}$, then $u$ is smooth inside $B_{\sigma / 2}\left(x_{0}\right)$.

Proof of Corollary 1.2. Let $\bar{\epsilon}$ be a number to be determined and let $B_{\sigma}\left(x_{0}\right)$ be a geodesic ball, where $(1 / \sigma) \mu\left(B_{\sigma}\left(x_{0}\right)\right)<\bar{\epsilon}$. Assume that $\bar{\epsilon}<\epsilon_{0}$; our main theorem implies that $\left\{u_{k}\right\}$ converges strongly to $u$ in $W^{1,2}\left(B_{\sigma / 4}\left(x_{0}\right), N\right)$. Then it follows from this $W^{1,2}$ strong convergence and the fact that $\left\{u_{i}\right\}$ is a minimizing sequence that $u$ is stationary with respect to both the first and the second variation (see [4]) inside $B_{\sigma / 4}\left(x_{0}\right)$, hence $u: B_{\sigma / 4}\left(x_{0}\right) \rightarrow N$ is a stationary harmonic map. We note that

$$
\frac{4}{\sigma} \int_{B_{\sigma / 4}\left(x_{0}\right)}|d u|^{2} d V \leq \frac{4}{\sigma} \int_{B_{\sigma}\left(x_{0}\right)}|d u|^{2} d V \leq \frac{4}{\sigma} \mu\left(B_{\sigma}\left(x_{0}\right)\right) .
$$

Hence, assuming that $\bar{\epsilon}<(1 / 4) \epsilon_{3}$ and applying Bethuel's theorem, we know that $u$ is smooth inside $B_{\sigma / 8}\left(x_{0}\right)$. With such a choice of $\bar{\epsilon}$, we define

$$
\Sigma=\left\{x \in M \mid \lim _{\sigma \rightarrow 0} \frac{1}{\sigma} \mu\left(B_{\sigma}(x)\right) \geq \bar{\epsilon}\right\} .
$$

A standard covering argument (see [4]) then shows that $\Sigma$ is a closed set with finite 1-dimensional Hausdorff measure. Hence, we conclude that $u$ is a smooth harmonic map from $M \backslash \Sigma$ to $N$, where $\Sigma$ is a close set with $\mathscr{H}^{1}(\Sigma)<\infty$.

ACKNOWLEDGMENTS. The author wants to thank Professor Richard Schoen for suggesting this problem. The author also wants to thank the referees for careful reading of the original manuscript.

\section{REFERENCES}

[1] F. Bethuel, On the singular set of stationary harmonic maps, Manuscripta Math. 78 (1993), no. 4, 417-443.

[2] J. Eells and J. C. Wood, Restrictions on harmonic maps of surfaces, Topology 15 (1976), no. 3, 263-266.

[3] J. Sacks and K. Uhlenbeck, The existence of minimal immersions of 2-spheres, Ann. of Math. (2) 113 (1981), no. 1, 1-24.

[4] R. M. Schoen, Analytic aspects of the harmonic map problem, Seminar on Nonlinear Partial Differential Equations (California, 1983), Math. Sci. Res. Inst. Publ., vol. 2, Springer, New York, 1984, pp. 321-358.

[5] R. M. Schoen and K. Uhlenbeck, A regularity theory for harmonic maps, J. Differential Geom. 17 (1982), no. 2, 307-335.

[6] L. Simon, Theorems on Regularity and Singularity of Energy Minimizing Maps, Lectures in Mathematics ETH Zürich, Birkhäuser Verlag, Basel, 1996, based on lecture notes by Norbert Hungerbühler. 
[7] C. Wang, Bubble phenomena of certain Palais-Smale sequences from surfaces to general targets, Houston J. Math. 22 (1996), no. 3, 559-590.

[8] B. White, Infima of energy functionals in homotopy classes of mappings, J. Differential Geom. 23 (1986), no. 2, 127-142.

Pengzi Miao: Department of Mathematics, Stanford University, Palo Alto, CA 94305, USA

E-mail address: mpengzi@math.stanford.edu 


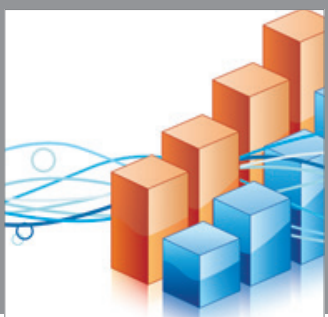

Advances in

Operations Research

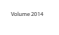

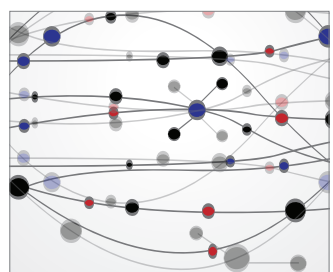

\section{The Scientific} World Journal
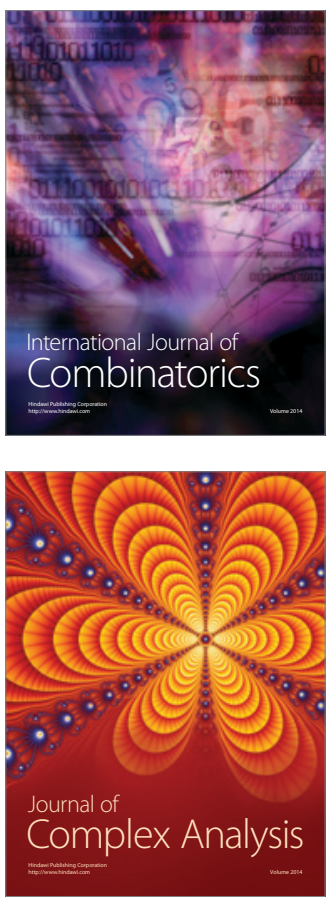

International Journal of

Mathematics and

Mathematical

Sciences
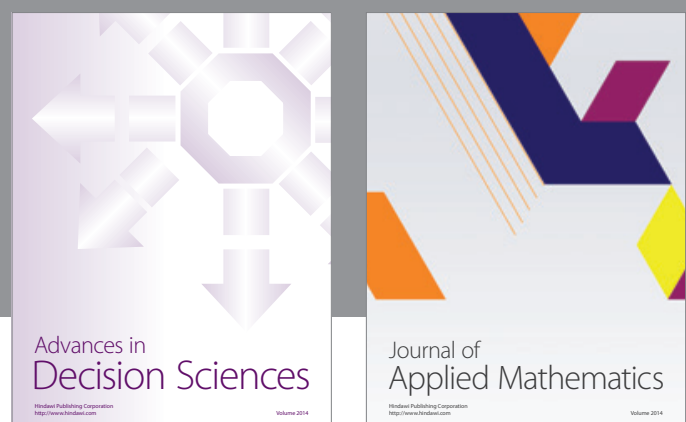

Journal of

Applied Mathematics
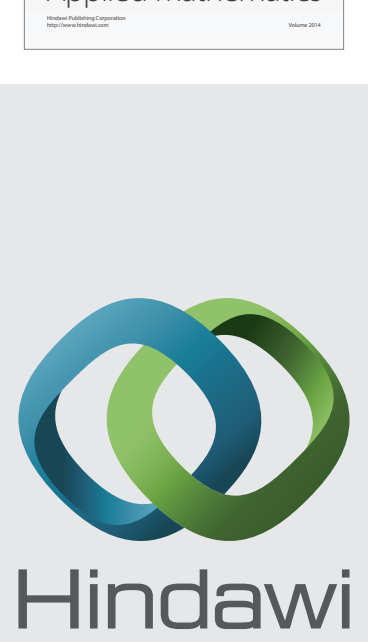

Submit your manuscripts at http://www.hindawi.com
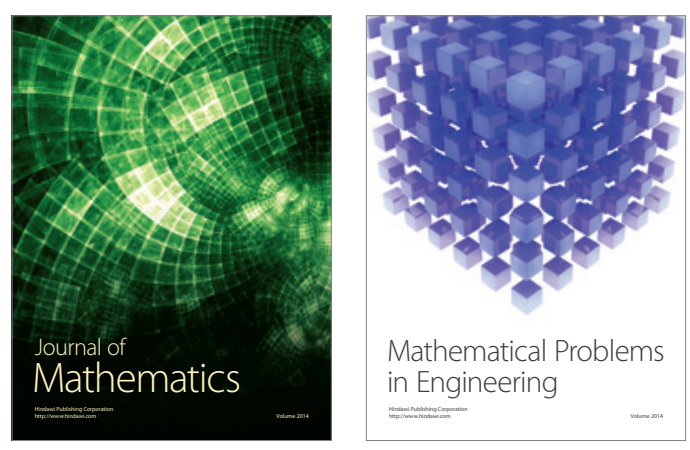

Mathematical Problems in Engineering
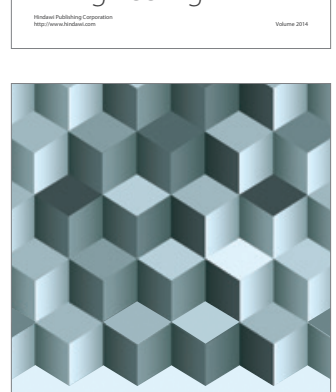

Journal of

Function Spaces
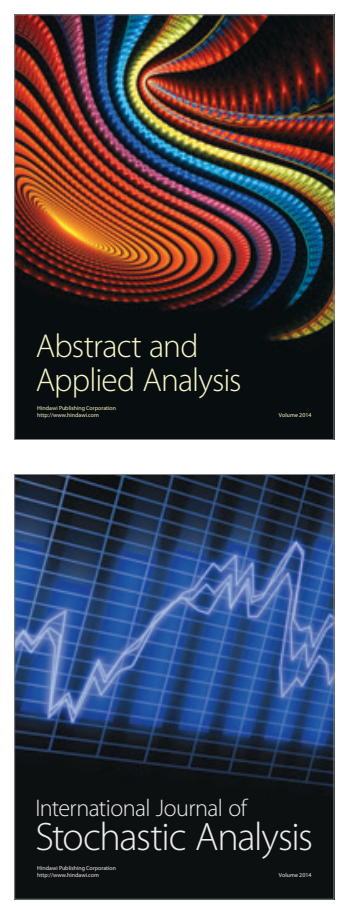

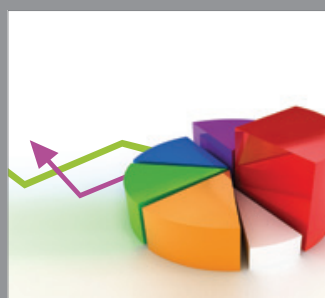

ournal of

Probability and Statistics

Promensencen
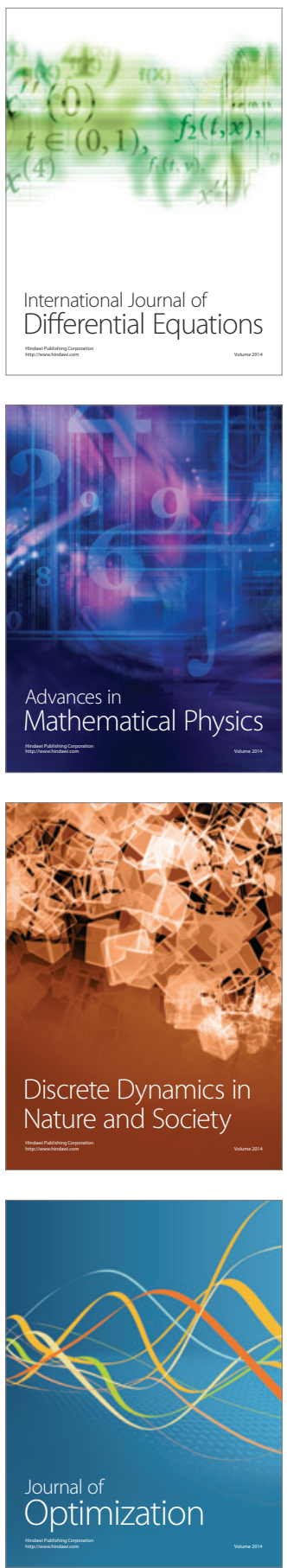